\title{
Marathi Translation and Linguistic Validation of an Updated European Organization for Research and Treatment of Cancer Quality of Life Module for Head and Neck
}

\author{
Chaitali M. Waghmare ${ }^{1}$ Hemant J. Pawar ${ }^{2}$ Vandana S. Jain ${ }^{1}$ Arya Bhanu ${ }^{1}$ Pradeep K. Thakur ${ }^{1}$ \\ Padmini H. Nirmal ${ }^{1}$
}

${ }^{1}$ Department of Radiation Oncology, RMC and PRH, PMTPIMS, Loni,

Address for correspondence Chaitali M. Waghmare, MD (RT), Maharashtra, India

${ }^{2}$ Department of Medical Statistics, PMTPIMS, PRH, Loni, Maharashtra, India \begin{abstract}
DNB, PGDCR, Department of Radiation Oncology, Rural Medical College and Pravara Rural Hospital, Pravara Medical Trust's Pravara Institute of Medical Sciences, Loni. Rahata, Ahmadnagar 413736, Maharashtra, India (e-mail: w.chaitali@gmail.com).
\end{abstract}

South Asian J Cancer 2021;9:199-203.

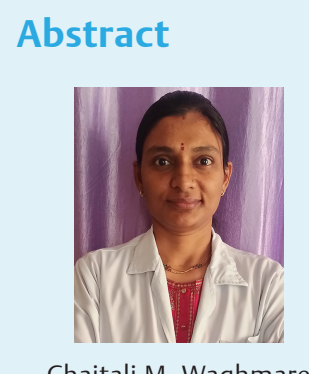

Chaitali M. Waghmare
Keywords

- European

Organization for

Research and

Treatment of Cancer

QLQ-H\&N43

- head and neck cancer

- Marathi translation

- quality of life
Aim This study was aimed to translate an updated European Organization for Research and Treatment of Cancer (EORTC) quality of life module for head and neck (EORTC QLQ-H\&N43) in grammatically and conceptually acceptable Marathi language and its linguistic validation.

Materials and Methods Approval was obtained from the Institutional Ethics Committee. The permission for translation was obtained from the EORTC translation unit (TU). The EORTC guidelines for the translation were followed to form a translation for pilot testing which was administered to 10 Marathi speaking head and neck squamous cell cancer (HNSCC) patients who gave informed written consent for the participation in the study. Patients were interviewed personally. The final Marathi translation was prepared and sent to EORTC TU for approval. Statistical analysis was performed using SYSTAT version 12 by Cranes software, Bengaluru, Karnataka, India.

Results After getting permission, the translation files were received from EORTC TU, including Marathi EORTC QLQ-H\&N35 for reference. Two forward translations, reconciled translation, back translations, first interim translation, translation for proof editing, and second interim translation (SIT) were prepared. This SIT was pilot tested in 10 Marathi-speaking HNSCC patients. Each patient was interviewed regarding difficulty in answering, confusing or offensive word, and reframing sentence. The questionnaire was well understood by patients reflecting its linguistic validity. After incorporating the changes as per the patient's interview, updated translation was prepared and sent to EORTC TU which was accepted and approved by EORTC. The psychometric analysis of pilot testing showed that the questionnaire is acceptable.

Conclusion Marathi translation of EORTC QLQ-H\&N43 is well accepted and understandable. It can be used for future studies.
DOI https://doi.org/10.1055/s-0041-1729444 ISSN 2278-330X

How to cite this article: Waghmare C. M, Pawar H. J, Jain V. S, Bhanu A, Thakur P. K, Nirmal P. H. Marathi Translation and linguistic Validation of an Updated European Organization for Research and Treatment of Cancer Quality of Life Module for Head and Neck. South Asian J Cancer 2021;9(4):199-203. (c) 2021. MedIntel Services Pvt Ltd.

MedIntel Services Pvt Ltd.. This is an open access article published by Thieme under the terms of the Creative Commons Attribution-NonDerivative-NonCommercial-License, permitting copying and reproduction so long as the original work is given appropriate credit. Contents may not be used for commercial purposes, or adapted, remixed, transformed or built upon. (https://creativecommons.org/licenses/by-nc-nd/4.0/). Thieme Medical and Scientific Publishers Private Ltd A-12, Second Floor, Sector -2, NOIDA -201301, India 


\section{Introduction}

In India, head and neck squamous cell cancer (HNSCC) is the second most common cause of cancer. ${ }^{1}$ The diagnosis of disease, disease itself, and its treatment affects the patient's social, psychological, and functional aspects. The advances in the multimodality treatment of HNSCC have translated into improvement in the locoregional control and overall survival probability. ${ }^{2}$ Hence, the quality of life (QOL) becomes important. Patient-reported outcome measure is an appropriate way to evaluate the QOL.

The European Organization for Research and Treatment of Cancer (European Organization for Research and Treatment of Cancer ) QOL module for head and neck cancer 35 (EORTC QLQ-H\&N35) is available for QOL study in patients with HNSCC. Its Marathi translation is available for use. ${ }^{3}$ An update of EORTC QLQ-H\&N35, that is, EORTC QLQ-H\&N 43 in English has recently completed the phase-IV study. ${ }^{4}$ As the module is in the English language and had to be self-administered, it cannot be used for all Marathi-speaking patients of HNSCC. Hence, the aim of this study was to formulate grammatically, linguistically, and conceptually acceptable, comprehensible, culturally acceptable and inoffensive Marathi translation of EORTC QLQ-H\&N43 which will be useful for Marathi-speaking patients of HNSCC of all educational levels.

\section{Materials and Methods}

\section{Part I: Pretranslation}

The Institutional Ethics Committee approval was obtained. A request was sent to the EORTC translation unit (TU) for permission to translate EORTC QLQ-H\&N43 in Marathi. EORTC translation procedure was followed.

\section{Part II: Translation}

The forward translation (FT) of the original English questionnaire was done by two independent native Marathi speakers who had good command over the English language. The translators had not seen this questionnaire before and they were primed with the aim of this study. EORTC QLQ-H\&N35 was given to them for reference. After discussion among the study coordinator and two FTs, reconciled translation was formed. This reconciled translation was then back translated in English by other two independent translators who were fluent in both languages. The first interim translation (FIT) was formed by comparing the back translations (BTs) with the original English tool and after adding appropriate modifications. This FIT was then sent to the EORTC office for discussion and comments. After all clarifications regarding translation with translation coordinator from EORTC TU, a translation for external proof editing was formed. Incorporating the corrections suggested by EORTC proof editor, second interim translation (SIT) for pilot testing was formed.

\section{Part III: Pilot Testing}

The Marathi translation for pilot testing i.e. SIT was given to literate Marathi-speaking and histopathology-proven nonmetastatic HNSCC patients, of all age group and either sex with Karnofsky's performance score of more than or equal to 70 , who had never seen these questionnaire before and gave informed written consent for the participation in the study. After completing the questionnaire, the translation was discussed individually with the patients. Patients were interviewed about the difficulty in understanding, difficulty in answering, and confusion regarding any question or word. They were also asked whether any question or word was objectionable or offensive. Patients were asked for their suggestions for framing a specific question or replacement of a specific relevant word. Incorporating the patient's suggestion, final Marathi translation was made and sent to the EORTC office for approval. Statistical analysis was performed using the statistical analysis software SYSTAT version 12 by Cranes Software, Bengaluru, Karnataka, India.

\section{Results}

\section{Part I: Pretranslation}

The permission to translate EORTC QLQ-H\&N43 was granted from EORTC TU. The translation files received from the EORC office included the translation manual, Excel copy of translation steps, and Marathi EORTC QLQ-H\&N35 for reference. The translation coordinators were assigned one from EORTC TU and the principal investigator (C.W.) from our team. The original EORTC QLQ-H\&N43 was compared with EORTC QLQ-H\&N43. The question numbers 31 to 33,35 to 39,41 to 45, and 60 and 61 of EORTC QLQ-H\&N43 were the same as in EORTC QLQ-H\&N35.

\section{Part II: Translation}

Two FTs of the original English questionnaire were received from FTs. Although EORTC QLQ-H\&N35 was given to them for reference, they suggested many changes in repeated questions along with the changes in instructions to fill the questionnaire. After the discussion among the study coordinator and two FTs, reconciled translation was formed.

\section{Reconciled Translation}

- Question number 73: FT1 was "Tumhala chawtana kahi tras janwat hota ka?" and FT2 was "Chawane awaghad watle hote ka?" We accepted FT2 for reconciled translation.

- The foot note stating "Sarva adhikar rakhun, Chawthya payritil aabhyasika" from FT2 was included in reconciled translation.

- For all other questions, FT1 was accepted with few corrections like the words "apnas/aaplya" were used in place of "tumhala/tumchya" which was showing more respect for the patients.

- Although the instructions for EORTC QLQ-H\&N35 were the same as EORTC QLQ-H\&N35, it was changed for the more respectful words for patients like "apnas" in place of "tumchya." The word "Gelya aathawdyat" was changed to "Gelya aathawadabharat" as per FT1. Question numbers 31 to 33 and 41 to 45 were accepted as it is in both FT1 and FT2 but in reconciled translation, the words "tumhala/tumchya" were replaced with "apnas/aaplya." 
This reconciled translation was then back translated in English by two other independent translators who were fluent in both the languages and had not seen the original English questionnaire. The FIT was formed by comparing the two BTs with the original English tool. BT1 and BT2 were comparable and giving same meaning as the original English questionnaire. Hence, the reconciled translation was used as FIT and was sent to EORTC TU for discussion and comments. Incorporating the suggestions and after explaining the queries from EORTC TU, Marathi translation was prepared for external proof editing. The following were the suggestions and explanations for EORTC TU queries.

Translation for external proof editing is as follows:

- Instruction: "Krupaya yogya aakdyabhowati vartul karun aaple uttar dyave" was changed to "Krupaya aapnasathi yogya aslelya aakdyabhowati vartul karun aaple uttar dyave."

- Question 33: clarification was given for the word soreness versus ulcers.

- Question number 51: the meaning in BT was confirmed for "problems with eating" versus "problems while eating." Furthermore, the meaning of word "Giltana" was confirmed as swallowing and not ingestion.

Incorporating the corrections suggested by external proof editor appointed by EORTC TU, SIT for pilot testing was formed. Postexternal proof editing changes were as follows:

- Instructions: "Barechda rugna tyana khalil pramane tras hot aahe kiwa khalil lakshananpaiki kahi lakshne disat aahet, ase sangat astat" was edited as "Kadhikadhi rugna tyana khalil pramane lakshne disat aahet kiwa samasya aahet, ase sangat astat."

- Instruction: "Krupaya aaplyasathi yogya aslelya aakdyabhowati vartul karun aaple uttar dyave" was edited as "Krupaya aapnasathi sarvat jast lagu asnarya aakdyabhowati vartul karun aaple uttar dyave."

- Instruction: "Jarase/thode" was edited as "jarase." The alternative word was removed.

- Question number 41, 44 to 47, 52 to 56, and 58 and 59: the part of the sentence "Adchan aali hoti ka?" was edited as "samasya aalya hotya ka?."

- Question number 73: "Chawane awaghad wattle hote ka?" was edited as "Aapnas chawnyat samasya aalya hotya ka?."

This postedited translation was discussed with the study team and was accepted as SIT which was used for pilot testing.

\section{Part III: Pilot Testing}

The Marathi translation for pilot testing, before SIT, was given to literate Marathi speaking and histopathology-proven nonmetastatic HNSCC patients, of all age group and either sex with Karnofsky's performance score of more than or equal to 70 , who had never seen these questionnaire before and gave informed written consent for the participation in the study. The characteristics of patients are described in - Table 1. After completing the questionnaire, the translation was discussed individually with the patients. Patients were interviewed about difficulty in understanding, difficulty in answering, and confusion regarding any question or word. They were also asked whether any question or word was
Table 1 Characteristics of ten patients selected for the pilot study

\begin{tabular}{|l|l|}
\hline Characteristics of patients & $n=10$ \\
\hline $\begin{array}{l}\text { Age }(y) \\
\text { Median (minimum-maximum) }\end{array}$ & $57(33-78)$ \\
\hline Sex & 8 \\
\hline Male & 2 \\
\hline Female & \\
\hline Site & 6 \\
\hline Oral cavity & 1 \\
\hline Oropharynx & 2 \\
\hline Hypopharynx & 1 \\
\hline Larynx & \\
\hline Stage & 8 \\
\hline III & 2 \\
\hline IVA & \\
\hline Treatment & 5 \\
\hline Surgery followed by chemoradiotherapy & 5 \\
\hline Radical chemoradiotherapy & \\
\hline
\end{tabular}

objectionable or offensive. Patients were asked for their suggestions for framing a specific question or replacement of a specific relevant word.

Patient interview report is as follows:

- Difficulty in answering: none of the patient-reported difficulty in answering any question.

- Confusing:

- Question number 32: one patient reported confusion in word "jabda (jaw)." After explaining, he understood the difference between the jaw and mouth. All other patients understood the question and there was no confusion.

- Question number 46: five patients had confusion in understanding the question. They said that there will always be a problem during coughing. Both the forward and BTs were rechecked. The FT1 was accepted for proof editing. However, when rechecked with BT, we found FT2 was appropriate. The FT1 meant "problem during coughing" and FT2 meant "problem with coughing." Hence, we accepted the FT2. The word "khoktanna" is replaced with the word "khoklyammule."

- Difficult words:

- Questions 35 to 37: two patients suggested, it would be easier to answer if we use the word "tras" in place of "samasya." However, the BT meant difficulty and not the problem as asked in the original questionnaire. Hence, the translation was not changed, as all the patents have answered and understood the question.

- Upsetting: none of the patients reported that any question was upsetting.

- Question numbers 60 and 61: although the question was not upsetting, six patients were shy during the interview and one patient refrained from answering the question. 
Rather seven patients said that the things should be explained to their partner that the disease is not contagious which is the common belief in the society.

- Alternative wording or suggestion:

- The alterative word was suggested for question numbers 35 to 37. However, it was not changed as explained earlier (tras vs. samasya).

- Question number 57: six patients pointed out that there is a repetition of the word "apnas." Hence, the second "apnas" in the sentence was deleted. "Apnas gongat aslelya watavarnat boltana samasya alya hoyta ka?."

None of the patients reported that the questions or any specific words used in the questionnaire were objectionable or offensive. The suggested corrections were incorporated and rechecked with BT. It was then sent to the EORTC TU. It was accepted and permission was granted for the future use of the final Marathi translation (Annexure 1).

The psychometric statistical analysis of pilot testing was performed using the statistical analysis software SYSTAT version 12 by Cranes Software, Bengaluru, Karnataka, India. The mean values of item scales are shown in -Figs. 1 and 2 , respectively. The internal consistency was measured to test the reliability of the translated questionnaire by calculating the Cronbach's $\alpha$. The Cronbach's $\alpha$ value item scales is given in - Table 2 . It was $>7$ (acceptable) for most of the functions/symptoms.

\section{Discussion}

The patient-reported QOL is a better measure of assessing the treatment outcome. EORTC QLQ-H\&N43 is a QOL module for HNSCC patients comprising 35 questions. Its update, that is, EORTC QLQ-H\&N43 contains 43 questions.

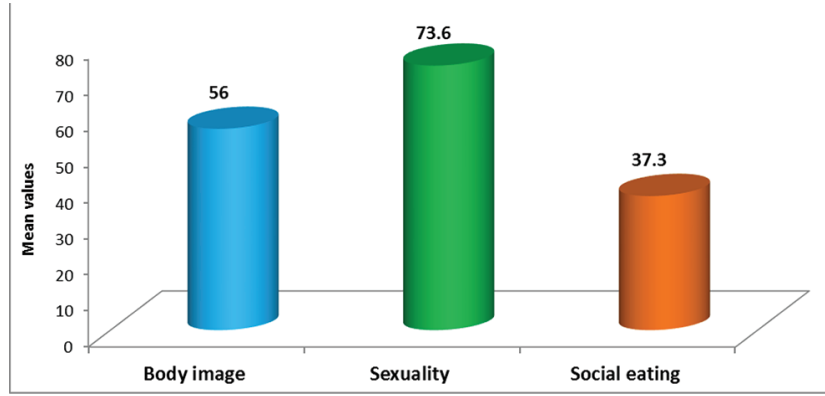

Fig. 1 Distribution of mean values of functional scale.

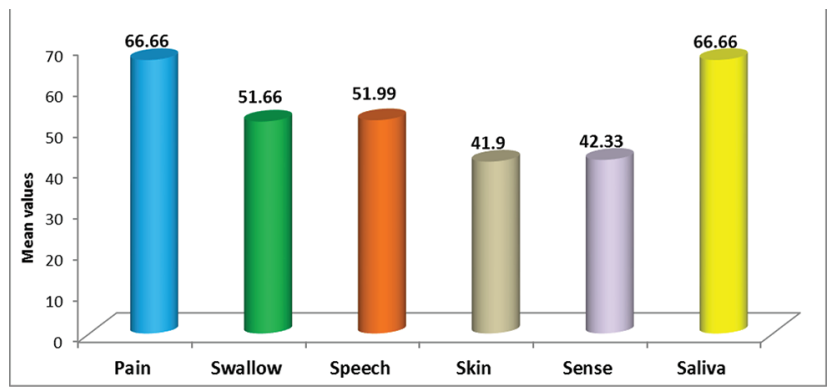

Fig. 2 Distribution of mean values of symptom scale.
Table 2 Multitrait scaling analysis with Cronbach's $\alpha$ between the scale items of European Organization for Research and Treatment for Cancer quality of life module for head and neck cancer 43

\begin{tabular}{|l|l|l|}
\hline Scale & Mean \pm SD & Cronbach's $\alpha$ \\
\hline $\begin{array}{l}\text { Body image (Q: 48, 49, } \\
\text { 50) }\end{array}$ & $56.00 \pm 13.72$ & 0.792 \\
\hline Sexuality (Q: 60, 61) & $37.30+/-10.43$ & 0.847 \\
\hline $\begin{array}{l}\text { Social eating } \\
\text { (Q:51,52,53,54) }\end{array}$ & $73.60+/-20.71$ & 0.816 \\
\hline Pain (Q: 31, 32, 33, 34) & $66.66 \pm 9.62$ & 0.912 \\
\hline $\begin{array}{l}\text { Swallow (Q: 35, 36, 37, } \\
\text { 38) }\end{array}$ & $51.66 \pm 12.30$ & 0.587 \\
\hline $\begin{array}{l}\text { Speech (Q: 47, 55, 56, } \\
\text { 57, 58) }\end{array}$ & $51.99 \pm 14.67$ & 0.622 \\
\hline Skin (Q: 65, 66, 67) & $41.90 \pm 8.44$ & 0.347 \\
\hline Sense (Q: 44, 45) & $42.33 \pm 8.60$ & 0.401 \\
\hline Saliva (Q: 42, 43) & $66.66 \pm 7.86$ & 0.774 \\
\hline
\end{tabular}

Abbreviation: Q, question; SD, standard deviation.

It assesses the functional (e.g., body image, sexuality, and social eating) and symptom scale (pain, swallow, speech, skin, sense, and saliva).

The question numbers 31 to 33,35 to 39,41 to 45 , and 60 and61 of EORTC QLQ-H\&N43 are the same as in EORTC QLQ-H\&N35. Although the questions were repeated and Marathi translation of EORTC QLQ-H\&N35 was used for reference, our translators had suggested few changes in it which were accepted by EORTC TU. The final Marathi translation of EORTC QLQ-H\&N43 was prepared by coordinated work between EORTC TU and the study team.

The patient's interview proved that the wordings were well and correctly understood reflecting the good linguistic validation. We found that few patients were shy or not comfortable in answering sex-related questions. We also came to know about the disbeliefs regarding HNSCC and sex which we tried to solve by communicating with the sexual partners of patients.

The psychometric analysis of pilot testing reflected that the questionnaire was acceptable for most of the subscales. The Cronbach's $\alpha$ value was less for few subscales. This can be because the study population was a mixed group of various head and neck subsites, for example, swallowing difficulty will be more in patients of cancer oropharynx and hypopharynx than the patients with carcinoma buccal mucosa. Similarly, it is the sense of smell.

\section{Conclusion}

Marathi translation of an updated EORTC QLQ-H\&N35 is well accepted and understandable. It is approved by the EORTC QOL group for future studies.

Financial Support and Sponsorship Nil. 
Conflict of Interest

There are no conflict of interest to declare.

Acknowledgments

Our sincere thanks to Dr Devidas Sitarampant Kulkarni and Dr. Padmini Nirmal for their help in the forward translation. We thank Dr. Arya Bhanu and Dr. Arnika Patil for their kind help in back translation. We are thankful to Mr. Vijay Pawar for his help in Marathi typing. We are thankful to Mr. Dogmara Kulis, the Translation Coordinator, EORTC QLQTU.

\section{References}

1 Bray F, Ferlay J, Soerjomataram I, Siegel RL, Torre LA, Jemal A. Global cancer statistics 2018: GLOBOCAN estimates of incidence and mortality worldwide for 36 cancers in 185 countries. CA Cancer J Clin 2018;68(6):394-424

2 Grégoire V, Langendijk JA, Nuyts S. Advances in radiotherapy for head and neck cancer. J Clin Oncol 2015;33(29):3277-3284

3 Chaukar DA, Das AK, Deshpande MS, et al. Quality of life of head and neck cancer patient: validation of the European organization for research and treatment of cancer QLQC30 and European organization for research and treatment of cancer QLQ-H\&N 35 in Indian patients. Indian J Cancer 2005;42(4):178-184

4 Singer S, Amdal CD, Hammerlid E, et al; EORTC Quality of Life and the EORTC Head and Neck Cancer Groups. International validation of the revised European Organisation for Research and Treatment of Cancer Head and Neck Cancer Module, the EORTC QLQ-HN43: phase IV. Head Neck 2019;41(6):1725-1737

\section{Best of ASCO India 2021 Conference}

$8^{\text {th }}$ to $11^{\text {th }}$ July 2021

Program Director - Dr M Vamshi Krishna, Director of Medical Oncology and BMT AIG Hospital, Hyderabad drmvkrishnaonco@gmail.com 995977112

Conference Managers - Kavina Creations

kashish@kavinacreations.com 9819025850 